\title{
Indicativos de Problemas de Comportamento e de Habilidades Sociais em Crianças: Um Estudo Longitudinal
}

\author{
Children's Behavior Problems and Social Skills: \\ A Longitudinal Study
}

\author{
Alessandra Turini Bolsoni-Silva" ${ }^{*}$, Edna Maria Marturano ${ }^{b}$, \\ \& Ludmilla Rubinger Bethonico Freiria ${ }^{a}$ \\ ${ }^{a}$ Universidade Estadual Paulista Júlio de Mesquita Filho, Bauru, Brasil \\ $\&{ }^{b}$ Universidade de São Paulo, Ribeirão Preto, Brasil
}

\begin{abstract}
Resumo
O estudo teve como objetivo avaliar o desenvolvimento de crianças indicadas por suas professoras na Educação Infantil (EI) como apresentando problemas de comportamento (Grupo IPC) ou comportamentos socialmente habilidosos (Grupo ICSH), em dois momentos: quando tinham cinco anos e quando tinham 10 anos. Participaram 48 professoras de 62 crianças de ambos os sexos. Os instrumentos utilizados foram Questionário de Respostas Socialmente Habilidosas para Professores e Escala Comportamental Infantil B. Os resultados indicaram diminuição dos problemas de comportamento e aumento dos comportamentos socialmente adequados no grupo IPC; os grupos eram bastante diferentes na primeira avaliação, quando na EI, mas as mudanças se atenuaram no ensino fundamental. Em ambas as avaliações, as crianças do Grupo ICSH foram avaliadas como mais habilidosas.

Palavras-chave: Habilidades sociais; Problemas de comportamento; Desenvolvimento infantil.

Abstract

The aim of this study was to assess the development of children whose primary teachers believed they had behavioral problems (IPC Group) compared to socially skilled children (ICSH Group). They were assessed in two occasions: when they were five years old and when they were 10 years old. Forty-eight teachers and 62 children of both genders participated in the study. The instruments used were the Teacher's Questionnaire for Socially Skilled Responses and the Child Behavior Scale B. In the results, the IPC Group showed a decrease of behavior problems and an increase in socially appropriate behavior. The groups were quite different in the first assessment, but their behaviors became more similar in elementary school. In both assessments, children in ICSH Group were assessed as more socially skilled.

Keywords: Social skills; Behavior problem; Child development.
\end{abstract}

A educação infantil é um importante recurso de apoio à criança, na construção de um repertório para enfrentar os desafios do ensino fundamental. A literatura destaca que a experiência escolar prévia favorece o ajustamento ao papel de estudante, influenciando positivamente os resultados escolares e o desenvolvimento da competência social (Benítez \& Flores, 2002; Entwisle \& Alexander, 1998; Trivellato-Ferreira \& Marturano, 2008).

Para algumas crianças, sobretudo para aquelas que têm na escola de educação infantil sua primeira experiência fora do círculo doméstico, o ingresso na educação infantil implica em mudanças profundas na sua vida diária. É difícil para o pré-escolar, por sua imaturidade cognitiva e de auto-regulação emocional, coordenar as demandas da escola relativas às regras, às tarefas e aos desafios da

* Endereço para correspondência: Av. Eng. Luiz Edmundo C. Coube, s/n, Vargem Limpa, Bauru, SP, Brasil, CEP 17015-970.E-mail: bolsoni@fc.unesp.br convivência em ambiente coletivo. A escola de educação infantil configura assim um contexto onde essas crianças serão mobilizadas por emoções intensas como excitação, ansiedade e medo (Graziano, Reavis, Keane, \& Calkins, 2007).

Situações corriqueiras como a disputa por brinquedos podem se tornar fontes de tensões cotidianas de difícil solução, já que as opções de coping do pré-escolar, ou seja, suas estratégias para gerenciar demandas internas e externas, percebidas como estressantes e avaliadas como sobrecarga aos recursos pessoais (Lisboa et al., 2002), são ainda limitadas a ações instrumentais, como busca do apoio dos cuidadores ou tentativa de mudar a situação aversiva, seja pela ação direta, seja pelo afastamento (Skinner \& Zimmer-Gembeck, 2007). Em um ambiente estranho, a busca de apoio é problemática, o afastamento pode levar ao isolamento e a ação direta frequentemente se manifesta em comportamentos impulsivos, como tomar o brinquedo ou empurrar o colega; tais comportamentos impulsivos, embora esperados nessa faixa etária, 
expõem a criança a repreensões dos adultos, aumentando seu desconforto. A ação do professor é importante na mediação de conflitos, podendo ser positiva, minimizando os riscos mencionados (Castro \& Bolsoni-Silva, 2008).

A adaptação à escola parece ser menos dramática para crianças com repertório mais desenvolvido de habilidades sociais, que segundo Del Prette e Del Prette (1999) "podem ser definidas como um constructo descritivo do conjunto de desempenhos apresentados pelo indivíduo diante das demandas de uma situação interpessoal" (p. 47); o termo é geralmente utilizado para designar um conjunto de comportamentos que envolvem interações sociais, abrangendo conceitos como assertividade (expressão apropriada de sentimentos negativos e defesa dos próprios direitos), habilidades de comunicação, de resolução de problemas interpessoais, de cooperação e de desempenhos interpessoais nas atividades ocupacionais (Del Prette \& Del Prette, 1999).

Estudos empíricos têm indicado que: (a) a ocorrência de problemas de comportamento é significativamente menor em pré-escolares com repertório mais desenvolvido de habilidades sociais (Keane \& Calkins, 2004); (b) o treino em habilidades sociais reduz agressão e problemas de comportamento em crianças (Sukhodolsky, Golub, Stone, \& Orban, 2005); (c) as crianças socialmente habilidosas estão mais protegidas contra vitimização física e relacional na educação infantil, ao passo que crianças com comportamentos problema estão mais expostas a ambos os tipos de vitimização (Garner \& Lemerise, 2007).

Sabe-se hoje que os desafios do ingresso na escola e o modo como as crianças os enfrentam podem influenciar a trajetória escolar dos alunos por vários anos. Estudos longitudinais sugerem efeitos persistentes de dificuldades comportamentais precoces, não apenas nos domínios do relacionamento com os pares (Ladd \& TroopGordon, 2003) e com o professor (Silver, Measelle, Armstrong, \& Essex, 2005), mas também no domínio do desempenho (Kwok, Hughes, \& Luo, 2007). Por outro lado, crianças com habilidades sociais mais desenvolvidas na educação infantil apresentam posteriormente menos problemas emocionais e de comportamento (Howes, Phillipsen, \& Peisner-Feinberg, 2000), melhor relacionamento com os professores e com os colegas (Howes et al., 2000; Ladd, Birch, \& Buhs, 1999) e melhor desempenho (Malecki \& Elliott, 2002). Desse modo, problemas de comportamento, entendidos como excessos ou déficits comportamentais que dificultariam o acesso da criança a novas contingências relevantes de aprendizagem, promotoras do desenvolvimento (Bolsoni-Silva \& Del Prette, 2003), constituem fator de risco quando identificados já na escola de educação infantil, por estarem associados à probabilidade aumentada de consequências negativas no desenvolvimento (Papalia, Olds, \& Feldman, 2006). Por outro lado, as habilidades sociais parecem funcionar como fatores de proteção, condições que reduzem impactos negativos na infância e prognosticam resultados positivos no desenvolvimento (Papalia et al., 2006), no sentido de promoverem ampliação de repertório, inibindo possíveis problemas advindos de comportamentos não adaptativos.

Manifestações externalizantes (por exemplo, fazer birra, bater) da fase pré-escolar têm sido consideradas como características transitórias do desenvolvimento normal, que tenderiam a diminuir com a idade (Bee, 2006). Sob essa ótica, o declínio nos comportamentos problema e sua substituição por formas mais maduras ou socialmente habilidosas de lidar com o ambiente seria uma tendência normativa no desenvolvimento. Pesquisas longitudinais (Howes et al., 2000; Welsh, Parke, Widaman, \& O’Neil, 2001) têm investigado essa tendência, encontrando resultados mistos, associados a indícios de estabilidade moderada nas diferenças individuais.

Nos anos pré-escolares, não se observam mudanças significativas, quer em comportamentos socialmente habilidosos, quer em comportamentos problemáticos (Howes et al., 2000; Keane \& Calkins, 2004). Na transição entre a educação infantil e o ensino fundamental, a evidência disponível de expansão nas habilidades sociais é positiva, se bem que restrita. Em um estudo do National Institute of Child Health and Human Development Early Child Care Research Network ([NICHD], 2003), as crianças mostraram aumento nas habilidades sociais, segundo avaliações concordantes das mães e dos professores, entre o jardim de infância e a $1^{a}$ série. Elas também se tornaram mais independentes, o que parece refletir uma tendência desenvolvimental de declínio na busca do apoio do adulto entre 5 e 7 anos de idade (Skinner \& Zimmer-Gembeck, 2007). Ao lado da ampliação do repertório, foi encontrada estabilidade nas diferenças individuais, expressa por uma correlação de 0,75 entre avaliações de habilidades sociais feitas pelas mães nos dois momentos e de 0,44 entre a avaliação feita pelos professores do jardim e da $1^{a}$ série.

Em relação ao curso do desenvolvimento de comportamentos problema, a literatura empírica apresenta resultados mistos. Côté, Vaillancourt, Barker, Nagin e Tremblay (2007) encontraram trajetórias declinantes de agressão física em $68 \%$ das crianças avaliadas por um cuidador primário aos 2 e aos 8 anos. Entretanto, 14,6\% mantiveram altos níveis de agressão ao longo de todo o período. No estudo do NICHD (2003), as mães relataram diminuição do comportamento externalizante entre o jardim de infância e a $1^{\mathrm{a}}$ série, mas os professores da $1^{\mathrm{a}}$ série apontaram mais problemas externalizantes que os do jardim de infância. Do ponto de vista cognitivo, a exposição ao ambiente escolar parece contribuir para a redução de soluções apoiadas na agressão (Borges \& Marturano, 2002).

A estabilidade dos problemas de comportamento se expressa em correlações moderadas entre medidas obtidas em dois momentos. Avaliações de professores do jardim de infância e da $1^{\text {a }}$ série mostraram correlação igual a 0,56 para problemas externalizantes (NICHD, 2003). 
Usando medidas compostas de avaliação do professor e dos colegas, Welsh et al. (2001) encontraram, entre a $1^{\text {a }}$ e a $3^{\mathrm{a}}$ série, correlação igual a 0,42 para comportamento agressivo. Ladd e Troop-Gordon (2003), baseados em avaliações dos professores no jardim de infância e na $4^{a}$ série, obtiveram indícios de correlação positiva entre agressão no jardim de infância e externalização na $4^{\text {a }}$ série $(r=0,57)$. Estabilidade moderada dos problemas de comportamento também se observa quando o informante é um cuidador primário (Anselmi et al., 2008; NICHD, 2003).

Ressalvadas as diferenças entre avaliadores, as correlações apenas moderadas, até mesmo entre avaliações feitas com um ano de intervalo (NICHD, 2003) - período relativamente curto para mudanças desenvolvimentais marcantes - apontam para a modificabilidade dos comportamentos, tanto os socialmente habilidosos como os problemáticos. Não obstante, a breve revisão da literatura aqui apresentada sugere um curso mais previsível para as habilidades sociais entre a educação infantil e o ensino fundamental, caracterizado por ampliação do repertório nos contextos familiar e escolar, provavelmente refletindo práticas de socialização compartilhadas na cultura (Chen \& French, 2008). Em relação aos comportamentos externalizantes, o cenário é aparentemente mais complexo, podendo apresentar maior variação entre contextos, assim como trajetórias divergentes, seja na direção de declínio ou de intensificação.

Ainda que haja uma tendência desenvolvimentista de atenuação dos problemas de comportamento (Anselmi et al., 2008; NICHD, 2003) e de expansão das habilidades sociais (Chen \& French, 2008), os estudos referenciados são consistentes quanto ao repertório de habilidades sociais, mas menos conclusivos quanto aos problemas de comportamento (Côté et al., 2007; NICHD, 2003; Silver et al., 2005). Na pesquisa de Côté et al. (2007), $14,6 \%$ das crianças mantiveram os problemas de comportamento externalizantes nas avaliações realizadas, sugerindo características de vulnerabilidade que implicam em maior tendência de manutenção dos problemas (Patterson, Reid, \& Dishion, 1992/2002).

Silver et al. (2005) verificaram que crianças com níveis extremos de externalização na educação infantil intensificavam essa tendência no ensino fundamental; no entanto, aquelas que tinham uma relação de proximidade afetiva com o professor da educação infantil reduziam significativamente os problemas de comportamento, em vez de aumentá-los.

Uma possível direção a partir da literatura da área, conforme explicitado acima é a de que haja uma tendência desenvolvimentista de atenuação dos problemas de comportamento, dependendo do contexto, e de expansão das habilidades sociais, dada a socialização das crianças. Ainda assim, há de se considerar que a vulnerabilidade das crianças com indicativos de problemas de comportamento possa interferir nessa tendência desenvolvimentista.
Portanto, entende-se que o acompanhamento do desenvolvimento de crianças quanto a indicativos de problemas de comportamento e habilidades sociais possa fornecer dados relevantes para a melhor compreensão das modificações de seu repertório comportamental em fases de transição escolar, bem como para a promoção de melhor qualidade de vida às crianças e aos adultos que lidam com elas, como os professores, tendo em vista que a escola é o segundo ambiente de socialização das crianças (Howes et al., 2000; Keane \& Calkins, 2004; Ladd et al., 1999). Considera-se relevante avaliar crianças identificadas, já na pré-escola, como tendo ou não indicativos de problemas de comportamento.

A presente pesquisa focaliza problemas de comportamento e comportamentos socialmente habilidosos de crianças em dois momentos de sua vida escolar - na Educação Infantil e no primeiro ciclo do Ensino Fundamental. Seu objetivo principal é o de comparar dois momentos distintos do desenvolvimento dos problemas de comportamento e das habilidades sociais de crianças indicadas pelas professoras da educação infantil como tendo ou não problemas de comportamento na escola. Considerando a tendência normativa do desenvolvimento (Bee, 2006), esperava-se expansão das habilidades sociais para a amostra como um todo; por outro lado, considerando a maior vulnerabilidade das crianças com problemas de comportamento, no sentido de retroalimentação das dificuldades pelas reações do ambiente (Patterson et al., 1992/2002), esperava-se a manutenção dos problemas de comportamento nesse grupo, sem diferença entre os dois momentos.

\section{Método}

\section{Modelo do Estudo}

Foi adotado um delineamento longitudinal de quatro anos. As crianças foram avaliadas duas vezes, na Educação Infantil e no primeiro ciclo do Ensino Fundamental.

\section{Participantes}

Participaram da pesquisa 62 crianças e suas professoras, em número de 48 , sendo 22 na Educação Infantil (EI) e 26 no Ensino Fundamental (EF). As crianças freqüentavam escolas públicas em uma cidade do centrooeste paulista, quando na EI; na segunda coleta freqüentavam escolas públicas, particulares e Serviço Social da Indústria (SESI). Foi critério de participação na pesquisa que a criança, quando freqüentava a EI, preenchesse uma dentre duas condições: (a) ter sido indicada pela professora como um aluno com comportamentos socialmente habilidosos (ICSH, $n=34$ ); (b) ter sido indicada pela professora como um aluno com problemas de comportamento (IPC, $n=28$ ). No Grupo ICSH havia 14 meninos e 20 meninas e no IPC, 14 meninos e 14 meninas. Na primeira avaliação, feita na EI, a idade média das crianças era 5,67 anos $(D P=0,59)$. Na segunda avaliação, feita no $\mathrm{EF}$, a idade média era 10,05 anos $(D P=$ 
0,80). As crianças nos grupos IPC e ICSH tinham idades equivalentes.

\section{Instrumentos}

Para avaliar comportamentos socialmente habilidosos foi utilizado o Questionário de Respostas Socialmente Habilidosas para Professores ([QRSH-PR], BolsoniSilva, Marturano, \& Loureiro, 2009). Para avaliar os problemas de comportamento foi empregada a Escala Comportamental Infantil B. de Rutter - Professores ([ECI-B], Santos, 2002).

O QRSH-PR consiste em uma lista de comportamentos socialmente habilidosos, constando de 24 itens, cada um com uma escala de três pontos $(2=$ se aplica; $1=$ se aplica em parte; 0 = não se aplica), cujos escores são somados, fornecendo o escore total da criança avaliada. Estudos sobre as propriedades psicométricas do instrumento foram realizados por Bolsoni-Silva et al. (2009) indicando consistência interna satisfatória e validade preditiva. $\mathrm{O}$ instrumento permite organizar os dados em três fatores: Sociabilidade e Expressividade Emocional (tem relações positivas, mostra interesse pelos outros, faz amigos, expressa frustração, comunica-se, expressa desejos, expressa direitos, expressa carinhos, brinca com colegas, usualmente fica de bom humor, faz elogios, cumprimenta, interage de forma não verbal, participa de grupos), Iniciativa Social (toma a palavra, expressa opiniões, participa de temas de discussão, toma iniciativas, negocia e convence, presta ajuda) e Busca de Suporte (procura atenção, faz pedido, faz perguntas).

A ECI-B consta de 26 itens descritivos de problemas de comportamento: (Impaciente/irrequieto, fica pouco tempo em uma atividade, destrói objetos, briga, irritável, desobediente, maltrata outras crianças, não é querido por outras crianças, fala mentiras, costuma roubar, mata aula, recusa-se a entrar na escola, parece tristonho, tende a ser fechado, fica facilmente preocupado(a), tem medo, queixa de dores de cabeça, é uma criança difícil, chupa dedos, rói unhas, apresenta tiques ou movimento repetitivo, gagueja, tem dificuldade de fala, tende a estar ausente da escola, molhou-se ou sujou-se na escola). Para cada item há uma escala de três pontos $(2=$ se aplica; 1 $=$ se aplica em parte; $0=$ não se aplica), cujos escores também são somados. Caso a soma atinja ou supere o valor nove, há a indicação clínica para o atendimento psicológico. Neste estudo, além do escore total, foi obtido um escore de Comportamento Problema, baseado em sete itens: Irrequieto, Inquieto, Destrói Coisas, Briga com Outras Crianças, Desobediente, Irritável, Maltrata Outras Crianças. A consistência interna da medida de Comportamento Problema foi satisfatória, com alfa igual a 0,916 para os dados obtidos na EI e 0,836 para os dados obtidos no EF.

\section{Procedimentos}

O estudo foi realizado em 13 Escolas Municipais de Educação Infantil (EMEIs) e 25 escolas de Ensino Fun- damental (escolas municipais, estaduais, particulares e SESI). Para compor a amostra foram visitadas as EMEIs, após a autorização concedida pela Secretaria Municipal de Educação Infantil. Em seguida, foi solicitada à direção das EMEIs permissão para que suas professoras participassem da pesquisa. A cada professora, após a assinatura do Termo de Consentimento Livre e Esclarecido, solicitou-se que indicasse, entre seus alunos, os três que apresentavam mais problemas de comportamento e os três com maiores indicativos de comportamentos socialmente habilidosos. Os pais das crianças foram consultados e aqueles que concordaram com a participação de seus filhos na primeira coleta de dados assinaram o Termo de Consentimento Livre e Esclarecido. As professoras responderam, para cada criança participante, à ECI-B e ao QRSH-PR. Os questionários foram aplicados individualmente na forma de entrevista, de forma a maximizar a compreensão dos itens e garantir maior qualidade na coleta dos dados. A entrevistadora formulava as questões oralmente e registrava as respostas no protocolo do instrumento.

Quatro anos depois, foi feito um rastreamento para localização das crianças nas escolas de ensino fundamental. Seguiram-se os procedimentos de obtenção de autorização institucional, assinatura dos termos de consentimento pelos professores e pelos pais e entrevistas com os professores para a aplicação dos dois instrumentos. O trabalho foi aprovado pelo Comitê de Ética em Pesquisa da universidade a que está vinculado.

Para a análise estatística dos dados, foram convertidos em escores-Z os escores totais do QRSH-PR e da ECI-B, bem como os escores dos fatores Sociabilidade, Iniciativa e Busca de Suporte do QRSH-PR e o indicador de Comportamento Problema derivado da ECI-B. Uma vez confirmado o requisito de homogeneidade de variância das variáveis entre os grupos IPC e ICSH, foi realizada a Análise de Variância - ANOVA - com medidas repetidas, para verificar variações significativas entre os escores na EI e no EF, assim como diferenças entre os grupos nos padrões de variação.

Variações entre resultados obtidos na EI e no EF foram investigadas também separadamente nos grupos IPC e ICSH. Essa análise foi conduzida sobre os escores brutos - total do QRSH-PR, total da ECI-B, fatores Sociabilidade, Iniciativa e Busca de Suporte do QRSHPR, indicador de Comportamento Problema da ECI-B e itens específicos de ambos os instrumentos. As comparações entre escores obtidos nos dois momentos foram feitas por meio do teste $t$ de Student para amostras dependentes.

Para avaliar a estabilidade dos comportamentos socialmente habilidosos e dos problemas de comportamento, o coeficiente $r$ de Pearson foi calculado entre resultados obtidos na EI e no EF, separadamente para os grupos IPC e ICSH. As correlações foram obtidas sobre os escores brutos - total do QRSH-PR, total da ECI-B, fatores Sociabilidade, Iniciativa e Busca de Suporte do QRSH- 
PR, indicador de Comportamento Problema da ECI-B e itens específicos de ambos os instrumentos.

Considerou-se como resultados estatisticamente significativos os obtidos com valores de $p$ iguais ou inferiores a 0,05 .

\section{Resultados}

O requisito de homogeneidade de variância das variáveis entre os grupos foi preenchido por todas as variáveis, com exceção do indicador de Comportamento Problema. A ANOVA com medidas repetidas não detec- tou diferenças entre os escores obtidos na EI e no EF para a amostra como um todo, mas indicou diferenças significativas entre os grupos IPC e ICSH em quatro dentre as cinco medidas investigadas: total do QRSH-PR, total da ECI-B e fatores Sociabilidade e Iniciativa do QRSH-PR. Interações significativas grupo $\mathrm{x}$ tempo foram encontradas em todas as comparações. Nas medidas de comportamento socialmente habilidoso, as médias do Grupo IPC aumentaram, ao passo que as do Grupo ICSH diminuíram. Na medida de problema de comportamento ocorreu o inverso: a média do Grupo IPC diminuiu e a do Grupo ICSH aumentou. Os resultados podem ser vistos na Tabela 1 .

Tabela 1

Estatísticas Descritivas para Comportamentos Avaliados na Educação Infantil e no Ensino Fundamental

\begin{tabular}{lccccccc}
\hline & \multicolumn{2}{c}{ Grupo IPC } & \multicolumn{2}{c}{ Grupo ICSH } & \multicolumn{3}{c}{ Estatística F } \\
\cline { 2 - 8 } & $\begin{array}{c}\text { EI } \\
\text { Média }(D P)\end{array}$ & $\begin{array}{c}\text { EF } \\
\text { Média }(D P)\end{array}$ & $\begin{array}{c}\text { EI } \\
\text { Média }(D P)\end{array}$ & $\begin{array}{c}\text { EF } \\
\text { Média }(D P)\end{array}$ & tempo & grupo & $\begin{array}{c}\text { tempo } \\
\text { x grupo }\end{array}$ \\
\hline Fatores: & & & & & & & \\
Sociabilidade & $15,82(6,28)$ & $21,00(6,11)$ & $26,91(2,26)$ & $24,41(4,70)$ & 0,42 & $51,19^{* *}$ & $12,08^{* *}$ \\
Iniciativa & $5,46(4,42)$ & $7,89(4,28)$ & $9,88(2,72)$ & $8,38(3,87)$ & 0,24 & $7,23^{*}$ & $9,84^{* *}$ \\
Busca de Suporte & $3,75(2,19)$ & $4,32(2,18)$ & $5,03(1,52)$ & $3,88(2,21)$ & 0,22 & 0,33 & $6,79^{*}$ \\
Totais: & & & & & & & \\
QRSH-PR & $25,93(11,73)$ & $34,50(11,55)$ & $43,23(5,78)$ & $38,29(9,18)$ & 0,16 & $24,59^{* *}$ & $15,05^{* *}$ \\
ECI-B & $15,86(5,36)$ & $10,43(6,15)$ & $5,32(3,82)$ & $5,85(4,76)$ & 0,08 & $44,57^{* *}$ & $7,88^{*}$ \\
\hline
\end{tabular}

Nota. A ANOVA foi processada sobre os escores $z . * p<0,05 ; * * p<0,01$.

Observa-se assim, nos resultados da ANOVA, que, de acordo com a percepção das professoras, as crianças com problemas de comportamento na EI mostram uma evolução positiva no EF, apresentando, quatro anos depois, menos comportamentos problemáticos e habilidades sociais mais desenvolvidas; já as crianças socialmente habilidosas na EI mostram uma tendência inversa, ainda que mais fraca. Com isso, as diferenças entre os grupos se atenuam no EF. No entanto, parece que as crianças do Grupo IPC não alcançam o nível de funcionamento dos colegas do Grupo ICSH, persistindo com alguma desvantagem, particularmente no que se refere aos problemas de comportamento e às habilidades de sociabilidade e expressividade emocional.

Como a diferença entre grupos, detectada pela ANOVA, poderia ser devida apenas ao critério de composição dos grupos na EI, os escores dos grupos IPC e ICSH no EF foram comparados por meio do teste $t$ de Student para amostras independentes. Os resultados indicaram diferenças significativas no fator Sociabilidade $(t=-2,502$, $p=0,015)$ e no escore total da ECI-B $(t=3,337, p=$ $0,001)$, confirmando, assim, a manutenção das diferenças entre os grupos, nessas duas variáveis, quatro anos depois da avaliação feita na educação infantil. Resultado semelhante foi encontrado ao se compararem os escores dos grupos no indicador de Comportamento Problema no $\mathrm{EF}(t=2,776, p=0,001)$.

Nas Tabelas 2 a 4 se encontram os resultados das análises feitas separadamente para cada grupo. Por causa das dimensões da tabela, apenas os itens com algum resultado significativo foram incluídos.

A Tabela 2 apresenta os resultados relativos aos comportamentos socialmente habilidosos do Grupo IPC. Dos 24 itens que compõem o instrumento, treze estão na tabela, todos eles com médias significativamente mais altas no EF em relação à EI. A estabilidade dos comportamentos é baixa; apenas os itens expressa opiniões, presta ajuda, faz perguntas e o fator Busca de Suporte mostram correlação moderada entre os escores obtidos nos dois momentos.

Os resultados do Grupo IPC referentes aos problemas de comportamento se encontram na Tabela 3. As análises detectaram resultados significativos em nove dos 26 itens da ECI-B, seja em termos de variação (teste $t$ ) ou de estabilidade $(r)$ entre a EI e o EF.

A tendência geral é de redução dos problemas de comportamento, com exceção do item destrói coisas, que mostra média maior no EF. Os itens que mostram redução sinalizam problemas de comportamentos relacionados a 
Bolsoni-Silva, A. T., Marturano, E. M. \& Freiria, L. R. B. (2010). Indicativos de Problemas de Comportamento e de Habilidades Sociais em Crianças: Um Estudo Longitudinal.

Tabela 2

Habilidades Sociais do Grupo IPC: Itens com Resultados Significativos nas Correlações e/ou Comparações entre $\mathrm{EI}$ e EF

\begin{tabular}{|c|c|c|c|c|}
\hline \multirow{2}{*}{ Itens - QRSH-PR } & \multicolumn{2}{|c|}{ Médias } & \multirow{2}{*}{$t$} & \multirow{2}{*}{$r$} \\
\hline & EI & EF & & \\
\hline Tem relações positivas & 1,07 & 1,64 & $-2,92 * *$ & $-0,19$ \\
\hline Faz amigos & 1,25 & 1,57 & $-2,08^{*}$ & 0,18 \\
\hline Expressa frustração adequadamente & 0,79 & 1,36 & $-2,74 * *$ & 0,07 \\
\hline Comunica-se positivamente & 1,07 & 1,57 & $-2,75^{* *}$ & $-0,10$ \\
\hline Expressa direitos adequadamente & 0,93 & 1,46 & $-3,22 * *$ & 0,32 \\
\hline Brinca & 1,61 & 1,89 & $-2,29 *$ & $-0,04$ \\
\hline Cumprimenta & 0,79 & 1,46 & $-3,19 * *$ & 0,09 \\
\hline Interage não verbalmente & 1,29 & 1,71 & $-2,27 *$ & 0,09 \\
\hline Expressa opiniões & 1,07 & 1,46 & $-2,37 *$ & $0,44 *$ \\
\hline Participa de discussões & 0,64 & 1,21 & $-2,66^{*}$ & 0,16 \\
\hline Negocia & 0,82 & 1,39 & $-2,74 * *$ & 0,20 \\
\hline Presta ajuda & 1,00 & 1,36 & $-2,07 *$ & $0,41 *$ \\
\hline Faz perguntas & 1,11 & 1,54 & $-2,58 *$ & $0,42 *$ \\
\hline Fator Sociabilidade & 15,82 & 21,00 & $-3,363 * *$ & 0,137 \\
\hline Fator Iniciativa & 5,46 & 7,89 & $-2,638 * *$ & 0,373 \\
\hline Fator Busca de Suporte & 3,75 & 4,32 & $-1,279$ & $0,414 *$ \\
\hline Total do QRSH-PR & 25,93 & 34,50 & $-3,38 * *$ & 0,34 \\
\hline
\end{tabular}

Nota. Teste $t$ de Student para amostras dependentes e $r$ de Pearson. EI = Educação Infantil; EF = Ensino Fundamental. $* p \leq 0,05 ; * * p \leq 0,01$.

Tabela 3

Problemas de Comportamento do Grupo IPC: ITENS com Resultados Significativos nas Correlações e/ou Comparações entre EI e EF

\begin{tabular}{lrrrr}
\hline \multirow{2}{*}{ Itens - ECI-B } & \multicolumn{2}{c}{ Médias } & & \multirow{2}{*}{$t$} \\
\cline { 2 - 3 } & EI & EF & & $r$ \\
\hline Irrequieto, correndo... & 1,00 & 0,57 & $2,27^{*}$ & $0,38^{*}$ \\
"Mata" aula & 0,50 & 0,07 & $2,46^{*}$ & $-0,17$ \\
Retorce-se, inquieto & 1,00 & 0,61 & 2,02 & $0,39^{*}$ \\
Destrói coisas & 0,57 & 0,88 & $-2,37^{*}$ & 0,28 \\
Briga com outras crianças & 1,04 & 0,54 & $2,55^{*}$ & 0,28 \\
Ausente da escola por razões triviais & 0,39 & 0,14 & $2,26^{*}$ & $0,62^{* *}$ \\
Desobediente & 0,82 & 0,32 & $3,33^{* *}$ & $0,51^{* *}$ \\
Chorou na escola ou recusou-se a entrar & 0,50 & 0,04 & $2,93^{* *}$ & $-0,12$ \\
Maltrata outras crianças & 0,75 & 0,21 & $3,07^{* *}$ & 0,13 \\
Indicador de Comportamento Problema & 6,36 & 3,16 & $3,69^{* *}$ & $0,55^{* *}$ \\
Total da ECI-B & 15,86 & 10,43 & $3,89^{* *}$ & 0,18 \\
\hline
\end{tabular}

Nota. $* p \leq 0,05 ; * * \leq 0,01$.

impulsividade/hiperatividade (irrequieto, desobediente) e dificuldades no relacionamento com as outras crianças (briga, maltrata), assim como problemas de adaptação à escola ("mata" aula, ausente por razões triviais, chorou ou recusou-se a entrar). Diferenças individuais persistem no indicador de Comportamento Problema. Poucos itens mostram correlação entre a EI e o EF. Dentre estes, estar ausente da escola por razões triviais se destaca, por apre- sentar correlação mais elevada $(0,62)$ entre os dois momentos.

$\mathrm{Na}$ Tabela 4 estão reunidos os resultados significativos referentes ao grupo de crianças indicadas pelas professoras da EI como socialmente habilidosas. Dez itens do QCSH-PR e cinco da ECI-B apresentam algum resultado significativo, seja de variação, seja de estabilidade entre a educação infantil e o ensino fundamental. 
Tabela 4

Habilidades Sociais e Problemas de Comportamento do Grupo ICSH: Itens com Resultados Significativos nas Correlações e/ou Comparações entre EI e EF

\begin{tabular}{|c|c|c|c|c|}
\hline Itens & Média EI & Média EF & $t$ & $r$ \\
\hline QCSH - Expressa frustração adequadamente & 1,97 & 1,79 & $2,24 *$ & $0,59 * *$ \\
\hline Expressa desejos & 1,91 & 1,68 & $2,10 *$ & $0,35 *$ \\
\hline Tem bom humor & 2,00 & 1,76 & $2,26^{*}$ & - \\
\hline Faz elogios & 1,68 & 1,23 & $2,45^{*}$ & 0,13 \\
\hline Cumprimenta & 1,97 & 1,44 & $3,45 * *$ & $-0,11$ \\
\hline Expressa opiniões & 1,73 & 1,53 & 1,49 & $0,34^{*}$ \\
\hline Participa de discussões & 1,70 & 1,56 & 1,04 & $0,34^{*}$ \\
\hline Toma iniciativas & 1,79 & 1,29 & $2,97 * *$ & 0,18 \\
\hline Presta ajuda & 1,85 & 1,71 & 1,30 & $0,34^{*}$ \\
\hline Faz perguntas & 1,82 & 1,41 & $2,29 *$ & $-0,10$ \\
\hline Fator Sociabilidade & 26,91 & 24,41 & $3,71 * *$ & $0,55^{* *}$ \\
\hline Fator Iniciativa & 9,88 & 8,38 & $2,26^{*}$ & $0,35^{*}$ \\
\hline Fator Busca de Suporte & 5,03 & 3,88 & $2,45^{*}$ & $-0,10$ \\
\hline Total do QRSH-PR & 43,23 & 38,29 & $3,40 * *$ & $0,43 *$ \\
\hline ECI - Irrequieto, correndo... & 0,26 & 0,23 & 0,27 & $0,40 *$ \\
\hline "Mata" aula & 0,18 & 0,15 & 0,44 & $0,64 * *$ \\
\hline Preocupado & 0,76 & 1,23 & $-2,68 *$ & 0,22 \\
\hline Ausente da escola por razões triviais & 0,26 & 0,26 & 0,00 & $0,56 * *$ \\
\hline Muito exigente & 1,26 & 0,82 & $2,04 *$ & $-0,02$ \\
\hline Total da ECI-B & 5,32 & 5,85 & $-0,70$ & $0,49 * *$ \\
\hline
\end{tabular}

Nota. $* p \leq 0,05 ; * * \leq 0,01$.

Confirmando os resultados obtidos com os escores totais apresentados na Tabela 1, os dados de variação indicam diminuição nas médias dos comportamentos socialmente habilidosos. Em relação aos problemas de comportamento, apenas dois mostram variação entre a EI e o EF: o item muito exigente diminui, ao passo que o item preocupado aumenta.

Contrastando com o Grupo IPC, o Grupo ICSH mostra continuidade nas diferenças individuais, indicada por uma correlação moderada entre as medidas globais obtidas na EI e no EF, tanto para os comportamentos problemáticos $(r=0,49)$ como para os socialmente habilidosos $(r=0,43)$, notadamente no fator Sociabilidade e Expressividade Emocional $(r=0,55)$. Por outro lado, o Grupo ICSH não apresenta correlação significativa entre as medidas de Comportamento Problema obtidas na EI e no EF, o que também o diferencia do Grupo IPC.

Outro ponto de contraste entre os grupos é o fator Busca de Suporte, que apresenta certa estabilidade no Grupo IPC (Tabela 2), mas não no Grupo ICSH. Os problemas de comportamento com maior estabilidade no Grupo ICSH estão relacionados a dificuldades de adaptação à escola: mata aula $(r=0,64)$ e ausente da escola por razões triviais $(r=0,56)$. Em relação a este último, também o Grupo IPC mostra continuidade nas diferenças individuais (Tabela 3).

\section{Discussão}

O principal objetivo deste estudo foi identificar o curso de desenvolvimento dos problemas de comportamento e das habilidades sociais de crianças indicadas pelas professoras da educação infantil como tendo ou não problemas de comportamento na escola. A previsão, baseada em evidência disponível na literatura, era de expansão das habilidades sociais para a amostra como um todo e persistência dos problemas de comportamento no grupo identificado com tais problemas pelas professoras da educação infantil. Os resultados confirmaram parcialmente o que era esperado e mostraram nuanças distintivas entre os grupos.

Quatro anos depois da indicação das professoras, os grupos ainda mantinham diferenças em habilidades sociais e problemas de comportamento, sugerindo persistência dos problemas no grupo IPC. No entanto, houve redução nos problemas de comportamento das crianças deste grupo na segunda avaliação, confirmando a visão de que os problemas de comportamento da fase pré-escolar tenderiam a diminuir com a idade (Bee, 2006).

Esse é um resultado instigante, tendo em vista achados de outras pesquisas que indicam manutenção ou mesmo agravamento dos problemas de comportamento, justamente nas crianças que precocemente exibem 
comportamentos problemáticos na escola (Howes et al., 2000, Keane \& Calkins, 2004, Ladd et al., 1999). No presente estudo, esse grupo supostamente mais vulnerável a processos de retroalimentação dos problemas de comportamento (Patterson et al., 1992/2002) manteveos, de fato, em níveis mais altos que os do Grupo ICSH, porém de forma atenuada na avaliação feita no ensino fundamental.

Esse achado sugere que as crianças indicadas como tendo problemas de comportamento na educação infantil tiveram trajetórias de desenvolvimento positivas, de acordo com a tendência de declínio nos comportamentos problema e sua substituição por formas mais maduras de lidar com o ambiente (NICHD, 2003), à medida que as crianças desenvolvem estratégias de auto-regulação emocional (Skinner \& Zimmer-Gembeck, 2007).

Essa interpretação é fortalecida quando os resultados de comportamentos problema são apreciados em conjunto com os resultados relativos às habilidades sociais. A expansão das habilidades sociais, esperada para a amostra como um todo, ocorreu apenas no Grupo IPC, sugerindo que as crianças desse grupo desenvolveram estratégias mais eficazes de lidar com os desafios da convivência. Esse resultado, a par com a redução em itens específicos da ECI relativos a agressões e brigas, confirma indícios prévios de que a exposição ao ambiente escolar contribui para a redução de soluções apoiadas na agressão (Borges \& Marturano, 2002).

Dos três fatores do QRSH-PR, o único que não mostrou aumento no Grupo IPC foi Busca de Suporte. A busca de suporte, indicando tentativas de obter ajuda, informação ou atenção, é um recurso importante para a adaptação da criança às demandas da sala de aula; no entanto, conforme assinalado na introdução, a estratégia de coping baseada na busca de suporte do adulto tende a declinar entre 5 e 7 anos de idade (Skinner \& ZimmerGembeck, 2007). Desse modo, o fato de as crianças do Grupo IPC não terem aumentado significativamente a busca de suporte, contrariando a tendência geral do grupo em direção a uma expansão das habilidades sociais, parece corresponder à tendência de desenvolvimento apontada na literatura. As crianças ampliam seu repertório de respostas socialmente habilidosas, exceto na categoria em que o esperado é uma diminuição com a idade.

A redução nos problemas de comportamento das crianças do grupo IPC, se por um lado diverge de alguns estudos, por outro lado é compatível com achados de pesquisas que têm detectado diferentes trajetórias para problemas de comportamento manifestados precocemente (Bongers, Koot, Ende, \& Verhulst, 2004; Côté et al., 2007; Silver et al., 2005). O estudo de Côté et al. (2007) sobre agressão mostra que a maior parte das crianças com alto nível de agressão física na fase préescolar tende a reduzir tais manifestações, ao passo que um pequeno grupo mostra persistência do alto nível inicial. Desse modo, o foco em diferentes cursos de desenvolvimento parece ser uma alternativa promissora para estudos sobre o curso de problemas de comportamento (Bongers et al., 2004). A diversidade nas trajetórias individuais a partir de um alto nível inicial de problemas de comportamento poderia explicar a correlação apenas moderada obtida no presente estudo, entre avaliações feitas nos dois momentos, um resultado muito semelhante aos encontrados em estudos anteriores (Ladd \& Troop-Gordon, 2003; NICHD, 2003).

O grupo ICSH também apresentou particularidades nos resultados. De acordo com a avaliação dos professores da educação infantil, o Grupo ICSH era composto por crianças mais habilidosas socialmente e que apresentavam menos problemas de comportamento. As avaliações feitas pelos professores do ensino fundamental tendem a confirmar essas características na comparação entre os grupos. Além disso, concordando com os achados de Howes et al. (2000), que encontraram estabilidade para o ajustamento social, na presente pesquisa se encontrou relativa estabilidade nas habilidades sociais do Grupo ICSH, em dois dos três fatores avaliados e no escore total do instrumento, ainda que tenha havido redução na segunda avaliação. A estabilidade moderada nas diferenças individuais em habilidades sociais foi expressa por uma correlação de 0,43 entre a avaliação feita pelos professores da EI e da $4^{\mathrm{a}}$ série, um resultado semelhante ao obtido no estudo do NICHD (2003).

A redução no nível de habilidades sociais entre as crianças do Grupo ICSH foi um resultado inesperado, dada a evidência de expansão dessas habilidades na transição entre a educação infantil e o ensino fundamental (NICHD, 2003) e a expectativa de um processo de socialização a partir da relação com os pares e professores (Borges \& Marturano, 2002). À falta de apoio na literatura, pode-se apenas especular a respeito de possíveis explicações para esse achado.

Uma explicação poderia estar nas diferenças entre os dois contextos educacionais em que as crianças foram avaliadas. Enquanto o projeto pedagógico da EI prevê mais atividades lúdicas, em que as crianças têm maior liberdade de interagir e se expressar, no EF se enfatiza o ensino de conteúdos e o enquadramento dos alunos em uma sala de aula com carteiras enfileiradas, onde se espera que permaneçam sentados. Nesse cenário, provavelmente o professor tem menos oportunidade de observar o repertório de comportamentos socialmente habilidosos dos alunos, já que estes se encontram mais contidos pela estrutura física e a natureza das atividades.

Outra explicação que pode ser lembrada estaria relacionada com o próprio desenho da pesquisa. Os grupos IPC e ICSH foram formados com base em dados fornecidos pelos professores da EI; cada um indicou, em sua turma, os três alunos que apresentavam mais problemas de comportamento e os três com maiores indicativos de comportamentos socialmente habilidosos. Essa condição pode ter induzido certo viés na avaliação dos professores da EI; eles podem ter-se inclinado inadvertidamente a 
realçar, em sua avaliação, as características que serviram de base para a indicação dos alunos socialmente habilidosos ou com problemas de comportamento.

No Grupo ICSH observou-se estabilidade das diferenças individuais em problemas de comportamento em três itens avaliados e no escore total da escala, ainda que os apresentassem em baixa frequência. Os resultados apresentados na Tabela 4 sugerem que dois itens com conteúdos semelhantes foram responsáveis pela estabilidade no escore total da ECI: "Mata" aula e Ausente da escola por razões triviais. Como esses itens não são representativos dos problemas mais comuns apresentados por escolares, não se pode afirmar, apenas com base nesses resultados, que tenha havido estabilidade dos problemas de comportamento no Grupo ICSH.

A ausência da escola por razões triviais foi também o item com maior estabilidade no Grupo IPC. Considerando a pouca idade das crianças, sobretudo na educação infantil, pode-se inferir que esses itens refletem principalmente a dificuldade da família para manter uma rotina regular de frequência da criança à escola, e não problemas da criança. Esses resultados não têm relação direta com os objetivos do estudo, porém vale destacálos pelo efeito negativo que tal condição pode ter no desempenho escolar, pois a estabilidade encontrada sugere que falhas precoces na supervisão parental tendem a persistir nos anos subsequentes da vida escolar da criança.

Deve-se considerar que os resultados deste estudo estiveram baseados no relato de professoras sobre o comportamento de seus alunos, ou seja, trata-se de uma avaliação indireta sobre os comportamentos elencados pelos instrumentos de pesquisa. Há, portanto, limitações quanto a ter garantia de que o relato tem correspondência direta com o comportamento avaliado. Por outro lado, nota-se convergência entre as avaliações feitas pelas professoras da EI e do EF, o que atesta confiabilidade dos dados obtidos. Estudos futuros poderiam valer-se de observação direta, além de medidas indiretas. Além disso, seria interessante a realização do acompanhamento do desenvolvimento a partir de relatos dos pais, de forma a verificar a consistência ou não dos resultados obtidos a partir do relato de professores.

\section{Considerações Finais}

Este trabalho aponta para uma tendência de os problemas de comportamento se atenuarem da Educação Infantil para o Ensino Fundamental. Com relação à evidência de aproximação entre os grupos inicialmente discriminados pelos professores como tendo ou não problemas de comportamento, este trabalho apresentou dados que demonstram que o Grupo IPC, classificado dessa forma na Educação Infantil, além de ter diminuído a manifestação de problemas de comportamento, também ampliou o repertório habilidoso aproximando-se do Grupo ICSH. Por outro lado, os resultados mostraram tam- bém que esse grupo continua em desvantagem. Essa constatação, associada ao importante achado referente à modificabilidade dos comportamentos, tanto os socialmente habilidosos como os problemáticos, tem implicações para ações educativas que assegurem aos alunos $o$ desenvolvimento de habilidades sociais, tendo em vista que estas reduzem as possibilidades de manifestação de problemas de comportamento.

\section{Referências}

Anselmi, L., Barros, F. C., Teodoro, M. L. M., Piccinini, C. A., Menezes, A. M. B., Araújo, C. L., et al. (2008). Continuity of behavioral and emotional problems from pre-school years to pre-adolescence in a developing country. Journal of Child Psychology and Psychiatry, 49, 499-507.

Bee, H. (2006). O ciclo vital (R. Garcez, Trad.). Porto Alegre, RS: Artmed.

Benítez, Y. G., \& Flores, S. M. (2002). Sondeo de habilidades preacadémicas en niños y niñas mexicanos de estrato socioeconômico bajo. Revista Interamericana de Psicología, 36(1/2), 255-277.

Bolsoni-Silva, A. T., \& Del Prette, A. (2003). Problemas de comportamento: Um panorama da área. Revista Brasileira de Terapia Comportamental e Cognitiva, 5(2), 91-103.

Bolsoni-Silva, A. T., Marturano, E. M., \& Loureiro, S. R. (2009). Contribuições para a construção e validação do Questionário de Respostas Socialmente Habilidosas: Versão para Professores - QRSH-PR. Spanish Journal of Psychology, 12, 349-359.

Bongers, I. L., Koot, H. M., Ende, J. V. D., \& Verhulst, F. C. (2004). Developmental trajectories of externalizing behaviors in childhood and adolescence. Child Development, 75, 1523-1537.

Borges, D. S. C., \& Marturano, E. M. (2002). Desenvolvendo habilidades de solução de problemas interpessoais no ensino fundamental. Paidéia, 12(24), 185-193.

Castro, A. B., \& Bolsoni-Silva, A. T. (2008). Habilidades sociais na educação: Relação entre concepções e práticas docentes na educação infantil. In V. L. M. F. Capellini \& R. M. Manzoni (Eds.), Políticas públicas, práticas pedagógicas e ensino-aprendizagem: Diferentes olhares sobre o processo educacional (pp. 296-311). São Paulo, SP: Cultura Acadêmica.

Chen, X., \& French, D. C. (2008). Children's social competence in cultural context. Annual Review of Psychology, 59, 591616.

Côté, S., Vaillancourt, T., Barker, E. D., Nagin, D., \& Tremblay, R. E. (2007). The joint development of physical and indirect aggression: Predictors of continuity and change during childhood. Development and Psychopathology, 19, 37-55.

Del Prette, Z. A. P., \& Del Prette, A. (1999). Psicologia das habilidades sociais: Terapia e educação. Petrópolis, RJ: Vozes.

Entwisle, D. R., \& Alexander, K. L. (1998). Facilitating the transition to first grade: The nature of transition and research on factors affecting it. The Elementary School Journal, 98, 351-365.

Garner, P. W., \& Lemerise, E. A. (2007). The roles of behavioral adjustment and conceptions of peers and emotions in preschool children's peer victimization. Development and Psychopathology, 19, 57-71. 
Graziano, P. A., Reavis, R. D., Keane, S. P., \& Calkins, S. D. (2007). The role of emotion regulation in children's early academic success. Journal of School Psychology, 45, 3-19.

Howes, C., Phillipsen, L. C., \& Peisner-Feinberg, E. (2000). The consistency of perceived teacher-child relashionships between preschool and kindergarten. Journal of School Psychology, 38, 113-132.

Keane, S. P., \& Calkins, S. D. (2004). Predicting kindergarten peer social status from toddler and preschool problem behavior. Journal of Abnormal Child Psychology, 32, 409415.

Kwok, O., Hughes, J. N., \& Luo, W. (2007). Role of resilient personality on lower achieving first grade students' current and future achievement. Journal of School Psychology, 45, 61-82.

Ladd, G. W., Birch, S. H., \& Buhs, E. S. (1999). Children's social and scholastic lives in kindergardner: Related spheres of influence? Child Development, 7, 1373-1400.

Ladd, G. W., \& Troop-Gordon, W. (2003). The role of chronic peer difficulties in the development of children's psychological adjustment problems. Child Development, 74, 1344-1367.

Lisboa, C., Koller, S., Ribas, F. F., Bitencourt, K., Oliveira, L., Porciúncula, L. P., et al. (2002). Estratégias de coping de crianças vítimas e não vítimas de violência doméstica. Psicologia: Reflexão e Crítica, 15, 345-362.

Malecki, C. K., \& Elliott, S. N. (2002). Children's social behaviors as predictors of academic achievement: A longitudinal analysis. School Psychology Quarterly, 17, 1-23.

National Institute of Child Health and Human Development Early Child Care Research Network. (2003). Social functioning in first grade: Associations with earlier home and child care predictors and with current classroom experiences. Child Development, 74, 1639-1662.

Papalia, D. E., Olds, S. W., \& Feldman, R. D. (2006). Desenvolvimento humano (8. ed., D. Bueno, Trad.). Porto Alegre, RS: Artmed.

Patterson, G., Reid, J., \& Dishion, T. (2002). Antisocial boys. Comportamento anti-social. Santo André, SP: ESETec (Original publicado em 1992)

Santos, P. L. (2002). Riscos e recursos em crianças com alto e baixo rendimento acadêmico: Um estudo comparativo. Tese de Doutorado não-publicada, Curso de Pós-Graduação em Psicologia, Universidade de São Paulo, Ribeirão Preto, SP.

Silver, R. B., Measelle, J. R., Armstrong, J. M., \& Essex, M. J. (2005). Trajectories of classroom externalizing behavior: Contribuitions of child characteristics, family characteristics, and the teacher-child relationship during the school transition. Journal of School Psychology, 43, 39-60.

Skinner, E. A., \& Zimmer-Gembeck, M. J. (2007). The development of coping. Annual Review of Psychology, 58, 119-144.

Sukhodolsky, D. G., Golub, A., Stone, E. C., \& Orban, L. (2005). Dismantling anger control training for children: A randomized pilot study of social problem-solving versus social skills training components. Behavior Therapy, 36, 15-23.

Trivellato-Ferreira, M. C., \& Marturano, E. M. (2008). Recursos da criança, da família e da escola predizem competência na transição da $1^{\mathrm{a}}$ série. Revista Interamericana de Psicología, 44, 407-410.

Welsh, M., Parke, R. D., Widaman, K., \& O`Neil, R. (2001). Linkages between children's social and academic competence: A longitudinal analysis. Journal of School Psychology, 39, 463-481. 